\title{
“ESTRATÉGIAS DE EDUCAÇÃO PERMANENTE EM SAÚDE NA REDE DE CUIDADOS À PESSOA COM DEFICIÊNCIA": AVALIAÇÃO DE ATIVIDADES DO PROJETO E CONTRIBUIÇÕES PARA AS PRÁTICAS COTIDIANAS DE TRABALHO E ASSISTÊNCIA
}

\author{
MOCELIN, G1 ${ }^{1}$, OLIVEIRA, B. R², SAUSEN, A. G. ${ }^{3}$, MAGEDANZ, M. C. ${ }^{4}$, DUBOW, C. ${ }^{5}$, GARCIA, E. L.6 ${ }^{6}$ WEIGELT, L. \\ D.7, KRUG, S. B. F. 8
}

PALAVRAS-CHAVE: Deficiência. Rede. Avaliação. Intersetorial. Ações.

\begin{abstract}
RESUMO
O objetivo do estudo foi avaliar atividades do projeto "Estratégias de Educação Permanente em Saúde na Rede de Cuidados à Pessoa com Deficiência" e analisar suas contribuições, a partir da ótica de seus participantes, para as práticas cotidianas de trabalho e assistência na rede de atenção às pessoas com deficiência (PcDs). 0 projeto interinstitucional foi desenvolvido nos anos de 2017 e 2018 na $28^{\text {a }}$ região de saúde do Rio Grande do Sul e contemplado no prêmio INOVASUS/2016 da Organização Pan-Americana de Saúde (OPAS) e Ministério da Saúde. Por meio de pesquisa exploratória e descritiva realizou-se, no presente estudo, a avaliação e contribuições das ações da segunda etapa do projeto. Os dados foram coletados por meio de um formulário, composto por seis blocos temáticos, contendo 29 questões fechadas e quatro questões abertas, durante a última oficina "Avaliação do Projeto e Criação do Grupo Condutor", realizada na Universidade de Santa Cruz do Sul, em que participaram 15 sujeitos, entre eles, profissionais da saúde, educação, assistência social e gestores da Rede de saúde de municípios da região. Os dados revelaram como produtivo e importante o desenvolvimento do projeto na região, sendo que os participantes, em sua maioria, avaliaram as ações muito positivamente e apontaram importantes contribuições para o aprimoramento de suas práticas de trabalho e assistência na rede de atenção às pessoas com deficiência, entre elas, maior clareza nos significados da deficiência, aprimoramento das ações de acolhimento dos PcDs e seus familiares nos serviços da rede de atenção, com articulação, fluxos e encaminhamentos mais claros e efetivos e também a sensibilização dos participantes para a temática, trazendo a importância de um olhar atento e menos excludente a esse segmento. Destacaram também o enfoque interdisciplinar, multiprofissional e interinstitucional do projeto como um dos fatores que contribuiu para o sucesso das ações.
\end{abstract}

\footnotetext{
${ }^{1}$ Graduado em Enfermagem pela Universidade de Santa Cruz do Sul, integrante do Grupo de Estudos e Pesquisas em Saúde (GEPS). Email: mocelinguilherme@gmail.com;

2Graduada em Enfermagem pela Universidade de Santa Cruz do Sul. E-mail: brendaraddatzz.br@gmail.com;

${ }^{3}$ Acadêmica do curso de Enfermagem, integrante do Grupo de Estudos e Pesquisas em Saúde (GEPS) e bolsista PUIC na Universidade de Santa Cruz do Sul. E-mail: anasausen98@gmail.com;

${ }^{4}$ Acadêmica do curso de Psicologia, integrante do Grupo de Estudos e Pesquisas em Saúde (GEPS) e bolsista PIBIC/CNPq na Universidade de Santa Cruz do Sul. E-mail: E-mail: carolmagedanz@gmail.com;

5 Doutoranda do Programa de Pós Graduação em Promoção da Saúde (PPGPS) e integrante do Grupo de Estudos e Pesquisas em Saúde (GEPS) na Universidade de Santa Cruz do Sul. E-mail: camiladubow@yahoo.com.br;

6 Doutora, docente do Departamento de Ciências da Saúde, Programa de Pós Graduação Mestrado Profissionalizante em Psicologia e Programa de Pós Graduação em Promoção da Saúde Mestrado e Doutorado na Universidade de Santa Cruz do Sul. E-mail: edna@unisc.br;;

${ }^{7}$ Doutora, docente do Departamento de Ciências da Saúde e do Programa de Pós Graduação em Promoção da Saúde (PPGPS) na Universidade de Santa Cruz do Sul. E-mail: lenid@unisc.br;

${ }^{8}$ Doutora, docente do Departamento de Ciências da Saúde e do Programa de Pós Graduação em Promoção da Saúde (PPGPS) na

Universidade de Santa Cruz do Sul. E-mail: skrug@unisc.br.
} 


\title{
"PERMANENT HEALTH EDUCATION STRATEGIES IN THE DISABILITY CARE NETWORK": EVALUATION OF PROJECT ACTIVITIES AND CONTRIBUTIONS TO DAILY WORKING PRACTICES AND ASSISTANCE
}

\author{
KEYWORDS: Disability. Network. Evaluation. Intersectoral. Actions.
}

\begin{abstract}
The aim of the study was to evaluate activities of the project "Strategies for Continuing Education in Health in the Network of Care for Persons with Disabilities" and to analyze their contributions, from the perspective of their participants, to the daily practices of work and assistance in the network of care for people with disabilities (PWD). The interinstitutional project was developed in 2017 and 2018 in the 28th health region of Rio Grande do Sul and was awarded in the INOVASUS/2016 award from the Pan American Health Organization (PAHO) and the Ministry of Health. Through exploratory e descriptive the evaluation and contributions of the actions of the second stage of the project were evaluated in the present study. Data were collected through a form composed of six thematic blocks, containing 29 closed questions and four open questions, and applied during the last workshop "Evaluation of the Project and Creation of the Driver Group", held at the University of Santa Cruz do Sul, in which 15 subjects participated, including health professionals, education, social assistance and managers of the Health Network of municipalities in the region. The data revealed how productive and important the development of the project in the region was, and the majority of the participants evaluated the actions very positively and pointed out important contributions to the improvement of their work practices and assistance in the network of care for people with disabilities, among them, greater clarity in the meanings of disability, improvement of the actions of welcoming PWD and their families in the services of the care network, with articulation, flows and more effective referrals and also the sensitization of participants to the theme, bringing the importance of a close and less exclusionary look to this segment. They also highlighted the interdisciplinary, multiprofessional and interinstitutional approach of the project as one of the factors that contributed to the success of the actions.
\end{abstract}

\section{INTRODUÇÃo}

A partir do advento da construção do Sistema Único de Saúde (SUS) entende-se que houveram diversas transformações no que diz respeito às políticas de saúde e educação, entre elas, a superação da setorialidade e fragmentação dos conhecimentos que integram estes dois campos. Deste modo, desenvolver e promover métodos de trabalho intersetoriais favorece novos modos de atuação em prol da promoção da saúde da população em todos os espaços, especialmente no que diz respeito às pessoas com deficiência (DIAS et al., 2016).

Para Almeida Filho (2000) a intersetorialidade pode ser definida como uma "estratégia de interferência em problemáticas complexas" (p. 31) que se faz necessária para a compreensão dos contextos complexos e vulnerabilidades em que os sujeitos estão inseridos. Permite que os diálogos entre os setores que se articulam possam confluir e alinhar-se de maneira positiva e resolutiva, de modo organizado e objetivo, proporcionando processos de trabalho em equipes com atuação partindo de distintos campos de atuação de trabalho: profissionais da saúde, assistência social, educação, gestores, usuários e familiares. Dessa forma, os setores envolvidos devem considerar os mesmos objetivos e suas ações devem contemplar seus diferentes campos focais.

Nesta esteira do pensamento observa-se que ações e programas como o Programa Saúde nas Escolas (PSE) e os Núcleos de Apoio à Saúde da Família (NASF) desenvolvem ações por meio da intersetorialidade, o que 
proporciona a mescla dos setores de educação, saúde e assistência social. Tal convergência de setores corrobora para acareação das fragilidades e vulnerabilidades que embaraçam o desenvolvimento dos escolares na rede pública de ensino brasileiro, bem como de suas famílias e comunidade, tornando-os, dessa forma, programas intersetoriais (CAMPOS; SOUZA; MENDES, 2015).

Sob essa perspectiva ressalta-se a Rede de Cuidados à Pessoa com Deficiência (RCPcD) e sua relativa invisibilidade mediante as políticas assistenciais, tendo em vista que as ações, com elevada frequência, apenas a atravessam sem ser direcionadas com especificidade. A RCPcD foi instituída, no SUS através da Portaria $n^{\circ} 793$ de 24 de abril de 2012, com o intuito de "ampliar o acesso, qualificar o atendimento em saúde, promover a vinculação das $\mathrm{PCD}$ e suas famílias aos pontos de atenção [...] e garantir a articulação e a integração desses pontos nos territórios" (DUBOW; GARCIA; KRUG, 2018, p. 456). Assim, essa Política prevê a organização do cuidado à essa população de forma intersetorial, garantindo a articulação de diferentes setores em prol das ações desenvolvidas às PcD.

Alinhado às necessidades da $\mathrm{PcD}$ e, tomando por base que $15 \%$ da população mundial possui algum tipo de deficiência e no Brasil, este índice é de 23,9\% (IBGE, 2010), emerge o projeto "Estratégias de Educação Permanente em Saúde na Rede de Cuidados à Pessoa com Deficiência", desenvolvido nos anos de 2017 e 2018 na $28^{\text {a }}$ região de saúde do Rio Grande do Sul e contemplado no prêmio INOVASUS/2016 da Organização PanAmericana de Saúde (OPAS) e Ministério da Saúde. Buscou estabelecer formas de educação permanente com os profissionais envolvidos na RCPCD, qualificando e proporcionando novos formatos de cuidado aos que dele anseiam e atuam. Frente a isso, o projeto caracteriza-se como interinstitucional e intersetorial e buscou refletir e articular ações entre profissionais da saúde, assistência social, educação, gestores, usuários e familiares da Rede de Atenção à Pessoa com Deficiência.

A execução de projetos e ações voltadas à Pessoa com Deficiência $(P c D)$ é de fundamental importância para maior entendimento e compreensão de suas diferentes necessidades, visto que, historicamente, as iniciativas de cuidado e de atenção à essa população eram realizadas de maneira fragmentada e isolada, ou seja, de maneira não intersetorial, se mostrando ineficazes na garantia do cuidado eficaz (CAMPOS; SOUZA; MENDES, 2015).

Maranhão (2019) destaca que o desenvolvimento de ações e projetos, no campo das políticas públicas, necessitam seguir alguns norteadores específicos que preconizam sua organização: construção, formulação, implementação, gestão, avaliação e controle social. Entende-se, por meio dessa sequência norteadora da formulação de projetos, a avaliação como uma das etapas que permite a reflexão das ações exitosas, bem como, das ações que não atingiram resultados esperados. Desta forma, a partir da avaliação pode-se repensar a dinâmica e aplicabilidade das ações, bem como dos recursos, permitindo a reformulação, se necessária, de uma ou mais etapas anteriores, como já observado, facilitando o alcance dos objetivos e, assim a qualificação do cuidado, da educação e das ações sociais propostas em tese.

Neste contexto da importância da avaliação de ações e projetos, o presente estudo visa avaliar atividades do projeto "Estratégias de Educação Permanente em Saúde na Rede de Cuidados à Pessoa com Deficiência" e analisar suas contribuições, a partir da ótica de seus participantes, para as práticas cotidianas de trabalho e assistência na rede de atenção às pessoas com deficiência. 


\section{FUNDAMENTAÇÃO TEÓRICA}

O campo de atuação do profissional que trabalha com políticas públicas é permeado por problemas sociais complexos e, portanto, está diretamente ligado ao trabalho articulado entre setores distintos e profissionais provenientes de diversas áreas relacionadas aos setores de saúde, educação e assistência social, caracterizando, assim, ações intersetoriais. Dessa forma, ressalta-se que a confluência de diversos atores e setores propicia mais agilidade e melhor resultado na resolução das problemáticas, uma vez que o diálogo se faz presente e, com esse fator, a construção conjunta de formatos mais resolutivos e efetivos são arquitetadas. Atravessado por essa relação, tal processo produz modificações na própria equipe, nos processos de trabalho e no meio em que visam a ação, além de produzirem menor custo de execução (ORNELAS; TEIXEIRA, 2015; PERES; CARMO; GUIZARDI, 2017; GRIGOLO; SCHNEIDER, 2017).

Ainda acerca da intersetorialidade, a mesma também se caracteriza como um modo de gestão pois é uma das prerrogativas contemporâneas acerca das políticas públicas (GRIGOLO; SCHNEIDER, 2017). Sob a ótica da gestão, esse processo de intersetorialidade proporciona o surgimento de novas direções às políticas públicas centradas em ações sociais e ações afirmativas, buscando a diminuição das barreiras existentes na sociedade através de ações que considerem o sujeito em sua integralidade (BOURGUIGNON, 2001).

Além disso, programas que contemplam a intersetorialidade em seu plano de ação, prevêem estratégias de intervenção positiva entre equipes e profissionais a fim de proporcionar qualidade e resolutividade de problemáticas à sociedade, entendendo a saúde como um conjunto de fenômenos complexos que estão relacionados para além dos determinantes biológicos, como os determinantes sociais. Assim, o campo da saúde intersetorial é visto como um campo conciliador, capaz de abarcar diferentes saberes relacionados ao cuidado e necessidades de cada sujeito, entendendo a saúde de forma integral (ORNELAS; TEIXEIRA, 2015; PERES; GRIGOLO; SCHNEIDER, 2017; DIAS et al., 2016).

Entrelaçado a essa linha de pensamento compreende-se que a Política Nacional de Educação Permanente em Saúde, implementada pela Portaria $n^{\circ}$ 198/GM/MS de 13 de fevereiro de 2004, surge como uma estratégia para atender as demandas sociais em saúde, a fim de realizar mudanças no modelo assistencial com o intuito de educar profissionais para atuar de forma crítica, e assim, transformar a realidade em que atuam (HADDAD; ROSCHKE; DAVINI,1994; BRASIL, 2004). Sendo assim, faz-se indispensável a valorização dos espaços e o reconhecimento dos saberes de forma cientificizada em ambientes de trabalho, ou não, como forma de ações educativas permanentes, contribuindo positivamente com a intersetorialidade, compreendendo as demandas apresentadas e as utilizando como formas de aprendizado constante

(LEMOS, 2016; CAMPOS; MARQUES; CECCIM; SILVA, 2019). A educação permanente em saúde associada a intersetorialidade possibilita cuidado integral e minimiza às ações fragmentadas, permitindo ações resolutivas e pontuais aos que se inserem sob tal/tais olhar/ações, possibilitando o aperfeiçoamento e qualificação do contexto. Ocupando um lugar de protagonismo nas estruturas organizacionais de ação a saúde, permitindo que a construção da acessibilidade ocorra de forma efetiva (AMORIM; LIBERALI; MEDEIROS NETA, 2018).

0 processo de avaliação de ações desenvolvidas a partir de políticas públicas, programas e projetos sociais surge como uma área de produção de conhecimento a partir da metade dos anos 60 , antes disso ela não era uma atividade metódica, organizada e regular. Portanto, a avaliação de tais projetos é baseada na 
metodologia de investigação para avaliar se os objetivos de tais ações foram de fato alcançados. No processo de avaliação, não estão envolvidos apenas os métodos e instrumentos que direcionam este, mas também os tópicos que devem ser avaliados e a forma como se deve obter informações válidas de tais tópicos (SERAPIONI, 2016). Sob essa perspectiva, compreende-se que alguns elementos chave se fazem necessários durante esse processo, sendo eles: o levantamento de olhares críticos acerca dos planos e ações desenvolvidos, com a finalidade de proporcionar melhorias sociais, reformulando, aprimorando ou mantendo as estratégias usadas, de acordo com os resultados obtidos durante tal processo; considerar minuciosa e rigorosamente cada apontamento, sendo eles positivos ou negativos, em que tal ferramenta possibilita a participação da sociedade de forma ativa. Desse modo, é notório que a ferramenta avaliativa permite o beneficiamento aos que tomam decisões e formulam planejamentos, bem como aos que são afetados por tais decisões, tendo em vista os processos envolvidos. Ressalta-se a importância da avaliação de programas, das políticas públicas, visto que por meio desta, é possível verificar a qualidade das ações, bem como mensurar o alcance, a efetividade e o impacto dos programas e das políticas e, além disso, reorganizar as ações (BRASIL, 1998; MARANHÃO, 2019; SERAPIONI, 2016).

Segundo Meirelles (2004), a definição de redes pode ganhar várias formas, entretanto: "mais importante que definir é entendê-la como uma proposta democrática de realização do trabalho coletivo e de circulação do fluxo de informações, elementos essenciais para o processo cotidiano de transformação social". 0 auxílio prestado pelas redes pode se estabelecer de diferentes formas, apoio informacional, orientações, aconselhamentos, entre outros. (SLUZKI, 1997). A implicação de tais atores e a disposição em fazer uma política pública pluralista e produzida de forma ascendente, isto é, com a participação direta da sociedade, foram e são decisivas para que, de fato, a Rede de Cuidados à Saúde da Pessoa com Deficiência se consolide como conquista da cidadania brasileira (CAMPOS; SOUZA; MENDES, 2015).

Sendo assim, a RDPcD, instituída no ano de 2012, através da Portaria 793/2012 tem como objetivo proporcionar cuidados à saúde das PcDs, especialmente nas áreas de promoção da saúde, identificação precoce de deficiências, prevenção de agravos, tratamento e reabilitação. Portanto, procura expandir o acesso e melhorar a qualidade da assistência prestada às PcD, através da articulação de diferentes serviços e níveis de atenção para que dessa forma, o cuidado se dê de forma integral. A rede é composta pela Atenção Especializada em Reabilitação Auditiva, Física, Intelectual, Visual, Ostomia e em Múltiplas Deficiências; Atenção Básica e Atenção Hospitalar e de Urgência e Emergência, a fim de suprir as demandas destes sujeitos (DUBOW; GARCIA; KRUG, 2018).

\section{MATERIAIS E MÉTODOS}

Realizou-se um estudo do tipo exploratório e descritivo, elaborado a partir da avaliação final da segunda etapa do projeto "Estratégias de Educação Permanente em Saúde na Rede de Cuidados à Pessoa com Deficiência", desenvolvido nos anos de 2017 e 2018, pelo Grupo de Estudos e Pesquisa em Saúde (GEPS) da Universidade de Santa Cruz do Sul (UNISC).

0 projeto tinha por objetivo fomentar discussões, reflexões e debates, culminando em decisões que se transformem em práticas e ações em prol de mudança, transformação ou aprimoramento dos processos de trabalho em relação às Pessoas com Deficiência no Sistema Único de Saúde, com maior resolutividade, 
aceitação, compartilhamento entre os coletivos de trabalho, e destes com os usuários do sistema. Foi desenvolvido na $28^{\mathrm{a}}$ região de saúde do Estado do Rio Grande do Sul (RS), composta por 13 municípios e localizada na região central do Estado do RS, com um total de 343.858 habitantes (IBGE, 2014). 0 RS tem um índice de $23,8 \%$ da população com algum tipo de deficiência, sendo que na $28^{\mathrm{a}}$ região de saúde este índice é de 30,81\% (IBGE, 2010).

0 projeto desenvolveu suas ações em duas grandes etapas. A primeira etapa, de cunho investigativo, consistiu na elaboração de um diagnóstico situacional e mapeamento da Rede de Cuidados à Pessoa com Deficiência e as ações de Educação Permanente em Saúde nos 13 municípios da $28^{\mathrm{a}}$ Região de Saúde/13a Coordenadoria Regional de Saúde do Estado do Rio Grande do Sul e contou com a participação de diversos atores envolvidos com a temática na região, entre eles, profissionais de saúde, gestores de serviços e PcDs. A partir das demandas educativas e de informações acerca da importância de se conhecer os sentidos da deficiência, a fim de auxiliar na compreensão das necessidades de saúde e das possibilidades de assistência em uma Rede de Cuidados da Pessoa com Deficiência apontadas pelos sujeitos na primeira etapa, elaborou-se as ações seguintes da segunda etapa do projeto, de cunho educativo, descritas a seguir.

Foram, então, realizadas dez oficinas de trabalho cooperativo com profissionais e gestores da saúde, da educação, assistência social, usuários e familiares, a partir da realização de um levantamento de necessidades com as equipes de saúde dos municípios e que contaram com a presença de 380 pessoas. As oficinas foram todas realizadas na UNISC e abordaram os seguintes temas: Política da Rede de Cuidados à Pessoa com Deficiência; Rede Intersetorial; Reabilitação Auditiva; Reabilitação Física; Políticas Públicas Voltadas para PCDs, Autismo; Tecnologia Assistiva; Projeto Terapêutico Singular e Inclusão Escolar.

Além das oficinas, também foram realizados dois eventos científicos, que também contemplavam estratégias educativas e de informação voltadas aos profissionais da RCPcD. 0 primeiro deles "Fórum Nacional da Rede de Atenção à Saúde da Pessoa com Deficiência e IV Seminário Científico do Programa de Pós-Graduação em Promoção da Saúde" realizado em 2017, serviu como disparador das discussões, reflexões e ações de educação permanente em saúde na rede de cuidados à PcD na região, contou com a presença de 611 participantes e envolveu mesas redondas e palestras. 0 segundo evento realizado em 2018, discutiu a temática da pessoa com deficiência em uma abrangência para além da área da saúde, envolvendo assistência social e educação e denominou-se: "Seminário Estadual de Atenção à Pessoa com Deficiência", contando com a participação de 172 pessoas e envolvendo mesas redondas e palestras.

Da mesma forma, foram elaborados materiais educativos com a intenção de auxiliar na organização dos fluxos assistenciais da Rede de Cuidados à Pessoa com Deficiência na região, entre eles, folders, banners, cartazes, documentários, cartilha do autismo, e-book intitulado "Rede de Cuidados à Pessoa com Deficiência: vivências, inovações e educação em saúde na $28^{a}$ Região de Saúde do Rio Grande do Sul".

0 presente artigo aborda a avaliação das ações da segunda etapa do projeto, compreendendo as nove oficinas anteriormente realizadas, os dois eventos científicos e os materiais educativos e foi realizada com 15 sujeitos, entre eles, profissionais da saúde, educação, assistência social e gestores da Rede, que participaram da décima e última oficina intitulada "Avaliação do Projeto e Criação do Grupo Condutor". 0 instrumento de coleta de dados foi um formulário de avaliação, distribuído durante essa oficina realizada na UNISC, composto por seis blocos temáticos contendo 29 questões fechadas e quatro questões abertas. As perguntas fechadas estavam 
categorizadas em cinco alternativas: ótimo, bom, regular, ruim e não sei/não participei. As questões abertas buscaram compreender a importância das atividades do projeto, percebida pelos participantes da pesquisa, para as suas práticas nos cotidianos dos serviços.

Para a análise de dados das questões fechadas, utilizou-se o Programa Excel para o compilamento dos dados, e após, estes foram analisados a partir de estatística descritiva, em frequências relativas e absolutas. A análise das questões abertas foi realizada por meio da Análise de Conteúdo de Bardin (2016). Após, a apresentação e discussão dos dados foi articulada em um verdadeiro mosaico, inter-relacionando os mesmos. Foram respeitados os preceitos éticos da Resolução 466/2012, com aprovação do projeto no Comitê de Ética da UNISC sob protocolo número 1.300 .666 e assinatura do Termo de Consentimento Livre e Esclarecido por parte dos integrantes do estudo. Para preservar a identidade dos participantes, as falas dos mesmos estão identificadas por F1, F2, F3, e assim por diante.

\section{RESULTADOS E DISCUSSÃO}

A seguir, os dados encontram-se apresentados e analisados, quantitativa e qualitativamente de forma conjunta, de modo a evidenciar a avaliação do projeto e suas contribuições para as práticas cotidianas de trabalho e assistência na rede de atenção às pessoas com deficiência.

Os resultados do presente estudo apontam que, em relação ao perfil dos participantes integrantes da décima oficina, a maioria eram mulheres $(n=14)$, com idades menor de 50 anos $(n=13)$ e faixa etária entre 41 a 50 anos ( $n=5)$. Os sujeitos atuavam nos municípios de Santa Cruz do Sul ( $n=08)$, Venâncio Aires ( $n=03)$, dois de Vera Cruz e dois de Passo do Sobrado. A área de atuação predominante foi a saúde, cujas áreas de atuação eram Enfermagem $(n=5)$, Assistência Social $(n=2)$, Educação Especial $(n=2)$, Fisioterapia $(n=2)$, Fonoaudiologia $(n=2)$ e Psicologia $(n=2)$.

Em relação à avaliação dos dois eventos, a maioria dos sujeitos considerou como ótimo ou bom as cinco categorias de análise: divulgação; programação; organização; temas abordados; conhecimento dos ministrantes, como é passível de se observar na Tabela 1.

TABELA 1 - Avaliação dos dois eventos científicos

\begin{tabular}{c|c|c|c|c} 
& \multicolumn{1}{c|}{$\begin{array}{c}\text { FÓRUM }(n= \\
15)\end{array}$} & $\%$ & SEMINÁRIO $(n=15)$ & $\%$ \\
\hline $\begin{array}{c}\text { DIVULGAÇÃO } \\
\text { Ótimo/Bom }\end{array}$ & 09 & 60 & 12 & 80 \\
\hline Regular/Ruim & 00 & 00 & 00 & 00 \\
\hline Não Participou & 06 & 40 & 03 & 20 \\
\hline TOTAL & 15 & 100 & 15 & 100 \\
\hline PROGRAMAÇÃO & 09 & 60 & 12 & 80 \\
\hline Ótimo/Bom & 00 & 00 & 00 & 00 \\
\hline Regular/Ruim & 06 & 40 & 03 & 20 \\
\hline Não Participou & 15 & 100 & 15 & 100 \\
\hline TOTAL & 09 & & \\
\hline
\end{tabular}




\begin{tabular}{|c|c|c|c|c|}
\hline \multicolumn{5}{|l|}{ ORGANIZAÇÃO } \\
\hline Ótimo/Bom & 09 & 60 & 12 & 80 \\
\hline Regular/Ruim & 00 & 00 & 00 & 00 \\
\hline Não Participou & 06 & 40 & 03 & 20 \\
\hline TOTAL & 15 & 100 & 15 & 100 \\
\hline \multicolumn{5}{|l|}{ TEMAS } \\
\hline Ótimo/Bom & 09 & 60 & 12 & 80 \\
\hline Regular/Ruim & 00 & 00 & 00 & 00 \\
\hline Não Participou & 06 & 40 & 03 & 20 \\
\hline TOTAL & 15 & 100 & 15 & 100 \\
\hline \multicolumn{5}{|c|}{ CONHECIMENTO MINISTRANTES } \\
\hline Ótimo/Bom & 09 & 60 & 12 & 80 \\
\hline Regular/Ruim & 00 & 00 & 00 & 00 \\
\hline Não Participou & 06 & 40 & 03 & 20 \\
\hline TOTAL & 15 & 100 & 15 & 100 \\
\hline
\end{tabular}

Fonte: dados da pesquisa, 2018.

A partir da tabela acima, identificou-se que, em relação ao Fórum, $60 \%$ dos sujeitos avaliou como ótimo/bom as cinco categorias de análise, enquanto no Seminário o percentual foi de $80 \%$ dos respondentes. Evidencia-se, ainda, que os resultados refletem a importância e a contribuição dos eventos para a região e para os profissionais, em relação ao contexto abordado.

Os relatos dos participantes sobre a avaliação dos eventos, em sua maioria, abordaram a importância dos mesmos de forma a refletir e esclarecer informações sobre o conceito de deficiência e as formas de assistência, ressaltando-se direitos, inclusão e políticas públicas voltadas aos PcDs. Também foram destacadas ações e estratégias de articulação da Rede de Cuidados e apontadas contribuições para o aprimoramento profissional individual no atendimento e assistência a $\mathrm{PcD}$, como pode ser observado a partir dos fragmentos de falas a seguir:

\footnotetext{
"O evento trouxe reflexões importantes para a minha atuação como profissional em meu município, principalmente quanto à (atenção) à saúde da $P c D$ e o quanto cada profissional deve estar implicado na qualificação do seu trabalho. Os palestrantes abordaram a atenção a $P C D$ de formas diferentes, levantando questionamentos quanto ao nosso entendimento do que é deficiência. Reforçando a importância dos participantes do projeto como multiplicadores das discussões, conceitos e informações desenvolvidas.". (F01).

“Os cuidados à pessoa com deficiência emergem no cotidiano das ESFs e rede de atenção, seja primária ou não, trabalho no município em forma de matriciamento e o escopo do fórum norteou formas de encaminhar, atender e articular a rede. Embora exija outras ações desde que trabalho na região foi o $1^{\circ}$ Evento desta parte que presenciei.". (F04).

“Movimentos de educação e saúde na região. Atualização e discussão sobre direitos, inclusão, cidadania, políticas públicas. Identificação das condições sociais, educacionais das PcDs na região e a existência da rede de Atenção. Excelente organização e temas condizentes com as questões e prioridades regionais". (F12.).
}

As estratégias de educação permanente são primordiais para qualificar a força de trabalho envolvida nos serviços de saúde da RCPCD, uma vez que, é um modelo estratégico que busca promover mudanças nos 
ambientes de atuação profissional, de maneira que os envolvidos atuem de forma crítica buscando modificar e transformar realidades, a partir de trocas de experiências e saberes no dia-a-dia, construindo novas formas de interação entre o serviço (trabalhadores) e a população (usuários). (LAVICH et al., 2017; CAMPOS; MARQUES; CECCIM; SILVA, 2019). De um modo geral, as evidências científicas apontam que os profissionais envolvidos na assistência às pessoas com deficiência encontram-se desqualificados o que acaba por favorecer o cuidado fragmentado, centrado no modelo biomédico, dificultando o desenvolvimento de ações efetivas e resolutivas voltadas à este público (FRANÇA et al., 2012).

Compreende-se que as atividades educativas são de primordial importância para a qualidade e efetividade dos serviços prestados na RCPCD pois através delas há a possibilidade de transformar práticas profissionais e de organização do trabalho envolvendo os diversos setores que compõem a Rede. Ainda, a Educação Permanente em Saúde (EPS) ao ser orientada para maior resolutividade de problemas de saúde das pessoas com deficiência a partir de momentos de reflexão e construção de conhecimentos, torna-se potencial para mudanças dos serviços de saúde, dos profissionais que neles atuam, bem como, dos próprios usuários, com a compreensão da necessidade de criar parcerias entre os setores que compõem a Rede e assim, facilitar a busca pelas transformações na mesma. (AMORIM; LIBERALI; MEDEIROS NETA, 2018; CAMPOS; MARQUES; CECCIM; SILVA, 2019).

Além disso, ações educativas voltadas também para profissionais dos setores de educação e assistência social possibilitam um olhar mais amplo para essa temática e, assim, a promoção de práticas alinhadas e contextualizadas acerca da realidade individual e social das PcDs. Engloba-se dessa forma, uma educação de qualidade e a efetivação dos direitos sociais dessa população, através da incorporação de novos saberes e práticas pautados na equidade e que visam a integralidade do sujeito, bem como a desconstrução de estereótipos e preconceitos ainda existentes na sociedade e que permeiam as práticas dos profissionais destes setores (TUON; CERETTA, 2017; HACK; CARVALHO, 2018).

Assim, acerca de práticas intersetoriais, que buscam um atendimento integral às PcDs, a pesquisa de Hack e Carvalho (2018) evidencia que a atenção às PcDs nos setores de saúde, educação e assistência social ainda são realizadas de forma setorializada e, além disso, não identificaram fatores importantes para o incentivo de práticas intersetoriais, como a comunicação, o planejamento, execução, monitoramento e avaliação integrados das ações nos diversos setores e serviços (HACK; CARVALHO, 2018).

Foi apontado também nos relatos dos integrantes do presente estudo, contribuições do projeto no sentido da necessidade de articular cuidados da rede aos familiares dos PcDs, destacando-se a situação de adoecimento e impossibilidade de trabalho dos mesmos, já que são responsáveis pelo cuidado diário do PcD:

\footnotetext{
"Sugiro talvez pensarmos em mais estratégias de trabalho e criação de novas Redes de Cuidados à Pessoa com Deficiência e também as famílias, que acabam muitas vezes fazendo um adoecimento ou mesmo não conseguem se inserir no mercado de trabalho". (F3).

“Muito importante para aprofundar discussões sobre a temática de Saúde da Pessoa com Deficiência. Possibilitou uma reflexão sobre a deficiência e seus impactos na vida das pessoas e suas famílias.". (F15).
}

Sob a perspectiva do cuidado da PcD no seio familiar, os autores Dantas et al., (2019) compreendem que estes processos repercutem diretamente nas ações, expressões e relações sociais que essas pessoas venham a 
desenvolver, tendo em vista o funcionamento familiar ser um dos fatores diretos de influência formadora das crianças, jovens, adultos e idosos com deficiência. Esse olhar sobre os cuidadores, além de merecer atenção tão especial e qualificada quando o cuidado despendido a PcD, é usualmente incumbida a progenitora, tarefa geradora de estresse, sobrecarga e impotência perante a situação, pois exige tempo de cuidado integral, dependendo da situação, o que impossibilita que a mesma ingresse/permaneça no mercado de trabalho, o que acaba impactando desfavoravelmente sua vida social.

Sob outro prisma, esse cuidado familiar é atravessado pelas necessidades de maiores conhecimentos e ações que proporcionem a formação desses cuidadores familiares às PcDs, o estudo de Marcon et al., (2007) reforça acerca das vantagens desse processo se dar em meio a família, tendo em vista, o cuidado tender a ser mais humanizado. Problematizando positivamente a temática e tornando os familiares protagonistas do cuidado, facilitando a construção de conhecimentos avançados para o manuseio de aparatos que se fazem necessário para a manutenção da qualidade de vida da pessoa em dependência, fator este, que se apresenta fragilizado (DANTAS et al., 2019), como foi possível perceber pelas explanações dos sujeitos do presente estudo.

Dessa forma, entender os impactos da deficiência na vida das famílias e/ou cuidadores pode contribuir com informações para os profissionais da saúde e da RCPcD, a fim de criarem estratégias que previnam agravos e adoecimentos à saúde destas pessoas, assim como, redes de apoio a estes familiares e da mesma forma, contribuir com o cuidado do profissional da saúde, tendo em vista que esse deve ser amplo e não apenas tratar aspectos clínicos da deficiência. Portanto, ressalta-se a importância de ações intersetoriais que garantam uma assistência integral para a PcD e sua família, tendo em vista que o cuidado não deve ser fragmentado e contemplar apenas os aspectos relacionados à deficiência (DANTAS et al., 2019).

Em relação as oficinas, a Tabela 2 apresenta dados referentes a participação dos sujeitos, bem como, a avaliação acerca da qualidade das mesmas.

TABELA 2 - Avaliação geral das oficinas

\begin{tabular}{|c|c|c|c|c|c|c|c|c|}
\hline OFICINAS & $\begin{array}{c}\text { ÓTIMO/B } \\
\text { OM }\end{array}$ & $\%$ & $\begin{array}{l}\text { REGULAR/R } \\
\text { UIM }\end{array}$ & $\%$ & $\begin{array}{l}\text { NÃO PARTICIPARAM/ } \\
\text { NÃO RESPODERAM }\end{array}$ & $\%$ & $\begin{array}{l}\text { TOTAL } \\
(n=15)\end{array}$ & $\%$ \\
\hline $1^{a}$ oficina & 07 & $\begin{array}{c}46 \\
7\end{array}$ & 00 & $\begin{array}{l}0 \\
0\end{array}$ & 08 & $\begin{array}{c}53, \\
3\end{array}$ & 15 & $\begin{array}{r}10 \\
0\end{array}$ \\
\hline $2^{a}$ oficina & 08 & $\begin{array}{c}53, \\
3\end{array}$ & 00 & $\begin{array}{l}0 \\
0\end{array}$ & 07 & $\begin{array}{c}46 \\
7\end{array}$ & 15 & $\begin{array}{c}10 \\
0\end{array}$ \\
\hline $3^{a}$ oficina & 07 & $\begin{array}{c}46 \\
7\end{array}$ & 00 & $\begin{array}{l}0 \\
0\end{array}$ & 08 & $\begin{array}{c}53 \\
3\end{array}$ & 15 & $\begin{array}{r}10 \\
0\end{array}$ \\
\hline $4^{a}$ oficina & 09 & 60 & 00 & $\begin{array}{l}0 \\
0\end{array}$ & 06 & 40 & 15 & $\begin{array}{l}10 \\
0\end{array}$ \\
\hline $5^{a}$ oficina & 09 & 60 & 00 & $\begin{array}{l}0 \\
0\end{array}$ & 06 & 40 & 15 & $\begin{array}{r}10 \\
0\end{array}$ \\
\hline $6^{a}$ oficina & 07 & $\begin{array}{c}46 \\
7\end{array}$ & 00 & $\begin{array}{l}0 \\
0\end{array}$ & 08 & $\begin{array}{c}53 \\
3\end{array}$ & 15 & $\begin{array}{c}10 \\
0\end{array}$ \\
\hline
\end{tabular}




\begin{tabular}{|c|c|c|c|c|c|c|c|c|}
\hline $7^{a}$ oficina & 06 & 40 & 00 & $\begin{array}{l}0 \\
0\end{array}$ & 09 & 60 & 15 & $\begin{array}{c}10 \\
0\end{array}$ \\
\hline $8^{a}$ oficina & 07 & $\begin{array}{c}46 \\
7\end{array}$ & 00 & $\begin{array}{l}0 \\
0\end{array}$ & 08 & $\begin{array}{c}53 \\
3\end{array}$ & 15 & $\begin{array}{c}10 \\
0\end{array}$ \\
\hline $9^{a}$ oficina & 11 & $\begin{array}{c}73 \\
3\end{array}$ & 00 & $\begin{array}{l}0 \\
0\end{array}$ & 04 & $\begin{array}{c}26 \\
7\end{array}$ & 15 & $\begin{array}{c}10 \\
0\end{array}$ \\
\hline
\end{tabular}

Fonte: dados da pesquisa, 2018.

A Tabela 2 aponta percentuais diferentes de avaliação como ótimo/bom, sendo necessário considerar que vários sujeitos não participaram de todas as oficinas e, consequentemente, não as avaliaram. Identifica-se que a nona oficina, com o tema Inclusão Escolar, obteve alta taxa de participação e avaliação positiva, considerando-se que, além dos profissionais da saúde e assistência social, houve também a participação de professores e gestores de educação da rede estadual e municipal da região, evidenciando que o tema da deficiência aborda vários campos do saber e do cuidado.

Por sua vez, a sétima oficina, intitulada Tecnologia Assistiva, contou com menor participação dos sujeitos e, consequentemente, com menos avaliações positivas. A Tecnologia Assistiva (TA), entendida como área do conhecimento interdisciplinar e enquanto terminologia ainda em construção, visa identificar recursos, métodos, estratégias, práticas e serviços que promovam maior independência, ampliação das habilidades funcionais e qualidade de vida de PcDs (SANTOS et al., 2017). Dessa forma, considera-se o pequeno número de participantes na oficina devido ao fato de tratar-se de um tema específico, técnico, ainda pouco conhecido, divulgado e compreendido como importante.

A Tabela 3 apresenta dados referentes a avaliação de quatro itens relacionados a todas as oficinas: temas; conhecimento dos ministrantes; foco nos processos de trabalho; formação de multiplicadores. As respostas dos sujeitos avaliaram, em sua grande maioria, como ótimos/bons a qualidade de todos eles.

TABELA 3 - Avaliação das oficinas por itens específicos

\begin{tabular}{|c|c|c|c|c|c|c|c|c|}
\hline & $\begin{array}{l}\text { ÓтIMO } \\
\text { /ВОМ }\end{array}$ & $\%$ & $\begin{array}{c}\text { REGULAR } \\
\text { / RUIM }\end{array}$ & $\%$ & $\begin{array}{c}\text { NÃO RESPONDEU } \\
\text { /NÃO SABE }\end{array}$ & $\%$ & TOTAL & $\%$ \\
\hline Temas & 13 & $\begin{array}{c}86 \\
6\end{array}$ & 00 & 0 & 2 & $\begin{array}{c}13,1 \\
4\end{array}$ & 15 & $\begin{array}{c}10 \\
0\end{array}$ \\
\hline $\begin{array}{l}\text { Conhecimentos } \\
\text { dos Ministrantes }\end{array}$ & 13 & $\begin{array}{c}86 \\
6\end{array}$ & 00 & $\begin{array}{l}0 \\
0\end{array}$ & 2 & $\begin{array}{c}13,1 \\
4\end{array}$ & 15 & $\begin{array}{c}10 \\
0\end{array}$ \\
\hline $\begin{array}{lr}\text { Foco } & \text { nos } \\
\text { Processos } & d e \\
\text { Trabalho } & \end{array}$ & 14 & $\begin{array}{c}93, \\
3\end{array}$ & 00 & $\begin{array}{l}0 \\
0\end{array}$ & 1 & 06,7 & 15 & $\begin{array}{c}10 \\
0\end{array}$ \\
\hline $\begin{array}{l}\text { Formação de } \\
\text { Multiplicadores }\end{array}$ & 14 & $\begin{array}{c}93 \\
3\end{array}$ & 00 & $\begin{array}{l}0 \\
0\end{array}$ & 1 & 06,7 & 15 & $\begin{array}{c}10 \\
0\end{array}$ \\
\hline
\end{tabular}

Fonte: dados da pesquisa, 2018 
Os dados apontam que as oficinas desenvolvidas durante o projeto tiveram importante contribuição como atividades educativas e de informação para profissionais, gestores, PcDs e suas famílias, pois foram fontes de esclarecimento acerca dos processos de trabalho com foco na assistência à $\mathrm{PcD}$, fluxos de encaminhamento na Rede de Atenção, conhecimentos sobre as deficiências, impactando dessa forma, na melhoria da assistência prestada a essas pessoas e suas famílias na região onde o projeto desenvolveu suas ações. Destaca-se ainda, a formação de multiplicadores como aspecto positivo a partir das nove oficinas desenvolvidas.

A exemplo disso, uma pesquisa realizada por Tedesco e Junges (2013) aponta a importância do desenvolvimento de ações educativas para o aperfeiçoamento e preparação dos profissionais atuantes na Rede, sendo que estes relataram se sentir desconfortáveis, ansiosos e angustiados no atendimento à usuários deficientes, pois sentiam-se despreparados e referiram a necessidade de atividades educativas para a melhoria da qualidade da assistência prestada. Pesquisa realizada por Alves; Ribeiro; Sampaio, (2016) demonstra que quando ações educativas são utilizadas dentro deste escopo, os resultados são satisfatórios.

Ainda sobre as oficinas, os relatos dos participantes, em sua maioria, abordam a contribuição das mesmas para informações e dados necessários para o seu cotidiano de trabalho, porém, desconhecidos por eles sobre itens como articulação, fluxos e encaminhamentos da Rede de Cuidados à PcD:

"O conhecimento levado e aprendido em cada oficina impacta na disseminação das informações e orientações para toda a atenção básica e especializada do município.". (F03).

"As oficinas impactaram positivamente no momento que oportunizaram discussões nas mais diversas áreas. É importante que se perceba a necessidade de trabalho em rede para divulgar, discutir e buscar direitos aos PcDs, dar visibilidade a estas pessoas.". (FO8).

“Assuntos como os abordados sempre nos sensibilizam, pois desconhecemos as legislações que amparam as pessoas com deficiência, seus direitos e se tratando de saúde, nem sempre sabemos para onde encaminhá-los". (F06).

Em consonância com as falas dos sujeitos do estudo e, em soma às ideias de Dubow, Garcia e Krug (2018), compreende-se que o conhecimento adequado e a apropriação correta dos fluxos da RCPcD possibilita a reflexão, planejamento e melhor encaminhamentos e atendimento aos usuários, por parte dos profissionais, conhecendo a realidade e qualificando a assistência. A sapiência acerca da $R C P c D$, não visa o conhecimento somente dos profissionais, em sua essência ela também inclui os usuários e seus familiares de forma ativa e participativa, tais ações abarcam e fomentam vínculos efetivos entre os serviços fortalecendo a intersetorialidade e às equipes circundantes nesse processo.

As oficinas também proporcionaram atualizações técnicas, de acolhimento e sensibilização a respeito de temáticas que envolvem a assistência à $\mathrm{PcD}$ e, consequentemente, do aprimoramento do trabalho individual e da equipe:

"Com as oficinas ampliei meu olhar no atendimento às pessoas com deficiência ao mesmo tempo conheci novos mecanismos de trabalho e de assistência. Como fazer encaminhamentos e conhecendo o trabalho da rede de cuidados.". (F12).

"Repensar práticas de intervenção e articulara Rede. Materiais disponibilizados pelo projeto e socializado com os municípios propôs uma ótima educação permanente de forma indireta dos que não puderam participar dos encontros.". (FO4).

"Através das oficinas foi possível uma maior sensibilização para a temática, tornando a questão mais presente no cotidiano dos serviços, possibilitando uma atenção mais humanizada e integral a estas pessoas.". (F15). 
É notório que para obter efetividade dos sistemas de saúde, educação e assistência, em especial no que se refere a RCPCD, um dos passos é qualificar a força de trabalho envolvida nos serviços oferecidos. Verifica-se contudo, que estudos e experiências envolvendo a EP na RCPcD ainda são incipientes e a temática é pouco investigada (ALVES; RIBEIRO; SAMPAIO, 2016). A exemplo disso, a criação de grupos focais em Núcleos de Apoios à Saúde da Família (NASF), em pesquisa realizada para analisar processos de cuidado de saúde de PcD e elaborar propostas de educação permanente, verificou-se que, a partir de reuniões semanais, a equipe relatou que as mesmas impactaram positivamente na resolução de problemas de PcDs assistidos, pois esclareceram processos de trabalho e permitiram aprimorar seus conhecimentos sobre o tema. Além disso, os trabalhadores reconheceram sua importância e necessidade sendo imprescindivel para o aperfeiçoamento e qualificação da Rede e dos profissionais que nela atuam (ALVES; RIBEIRO; SAMPAIO, 2016). Outra pesquisa como a de Maia, Pagliuca e Almeida (2014) demonstrou que os profissionais atuantes na Rede carecem de informações adequadas no que concerne às pessoas com deficiência o que acaba implicando desfavoravelmente na assistência prestada. Dessa forma, torna-se nítida a importância de qualificar a força de trabalho envolvida nos serviços oferecidos, para que os resultados da assistência prestada sejam satisfatórios.

A Tabela 4, a seguir, explicita os dados acerca da avaliação dos materiais educativos e de divulgação do projeto nos itens: organização; relevância dos conteúdos; clareza dos conteúdos; divulgação e distribuição.

TABELA 4 - Avaliação dos materiais educativos e de divulgação por itens específicos e por resultados

\begin{tabular}{|c|c|c|c|c|c|c|c|c|}
\hline & $\begin{array}{l}\text { ÓTIMO/B } \\
\text { OM }\end{array}$ & $\%$ & $\begin{array}{c}\text { REGULAR/R } \\
\text { UIM }\end{array}$ & $\%$ & $\begin{array}{c}\text { NÃO RESPONDEU/ } \\
\text { NÃO SABE }\end{array}$ & $\%$ & TOTAL & $\%$ \\
\hline Organização & 14 & 93,3 & 00 & 00 & 01 & $\begin{array}{c}06 \\
7\end{array}$ & 15 & $\begin{array}{r}10 \\
0\end{array}$ \\
\hline $\begin{array}{l}\text { Relevância } \\
\text { dos } \\
\text { Conteúdos }\end{array}$ & 14 & 93,3 & 00 & 00 & 01 & $\begin{array}{c}06 \\
7\end{array}$ & 15 & $\begin{array}{c}10 \\
0\end{array}$ \\
\hline $\begin{array}{l}\text { Clareza dos } \\
\text { Conteúdos }\end{array}$ & 14 & 93,3 & 00 & 00 & 01 & $\begin{array}{c}06 \\
7\end{array}$ & 15 & $\begin{array}{c}10 \\
0\end{array}$ \\
\hline $\begin{array}{l}\text { Divulgação e } \\
\text { Distribuição }\end{array}$ & 14 & 93,3 & 00 & 00 & 01 & $\begin{array}{c}06 \\
7\end{array}$ & 15 & $\begin{array}{r}10 \\
0\end{array}$ \\
\hline
\end{tabular}

Fonte: dados da pesquisa, 2018.

Os dados apresentados na tabela 4 acerca dos materiais pedagógicos, educativos e de divulgação produzidos pelo projeto foram avaliados como ótimo/bom pela maioria dos sujeitos (93,3\%). Considera-se, a partir disso, que os materiais elaborados de forma coletiva e em parceria intersetorial, características permanentes das ações do projeto, foram relevantes para o cotidiano de trabalho dos profissionais, ressaltando, assim, que atingiram sua finalidade, servindo de apoio técnico para profissionais, PcDs e familiares.

Foram elaborados pelos participantes do projeto, diversos materiais pedagógicos educativos de apoio para as ações em saúde voltadas às Pessoas com Deficiência, criado a partir das demandas elencadas pelo coletivo. Estes materiais tiveram como objetivo informar aos trabalhadores atuantes na rede, gestores, usuários e comunidade acadêmica sobre as ações e serviços oferecidos para as Pessoas com Deficiência pelo Sistema 
Único de Saúde da região. Ainda, objetivaram sensibilizar os trabalhadores para a ampliação do olhar no cuidado às pessoas com deficiência, almejando uma atenção mais integral e humanizada que promova a equidade na atenção à saúde destes usuários.

Em seus relatos, os participantes apontaram que a divulgação dos materiais educativos poderia ter sido melhor desenvolvida, porém, em sua maioria, referiram a importância desses materiais como estratégias de educação permanente, de divulgação da RCPCD e de apoio para os profissionais e usuários da rede:

\footnotetext{
“Repensar práticas de intervenção e articular Rede. Materiais disponibilizados pelo projeto e socializado com os municípios propôs uma ótima educação permanente de forma indireta dos que não puderam participar dos encontros.". (F04).

“Os materiais servem de apoio para pesquisa e divulgação aos usuários dos serviços onde atuamos [...]". (F14).

"Os materiais possibilitaram um maior conhecimento sobre a rede à pessoa com deficiência, além de uma maior divulgação desta, tanto para trabalhadores quanto para usuários desta rede.". (F15).
}

Levando em consideração os conceitos e características da educação permanente, tem-se nos materiais pedagógicos uma estratégia de desenvolvê-la nos diferentes setores que integram a RCPcD. A difusão de informações através deste tipo de abordagem garante aquisição de conhecimento técnico, de fácil acesso e compressão, bem como, agrega e colabora para a uniformização de orientações pertinentes à temática (ZOMBINI; PELICIONI, 2011). Além disso, sua elaboração contribui significativamente quando conciliado à outras ações de educação permanente, pois a partir deles pode-se perceber o que não foi compreendido em outras ações, por exemplo. Uma vez que sua elaboração apropria-se do conhecimento científico, os materiais pedagógicos compilam e selecionam informações pertinentes e necessárias, de fácil leitura e convidativa ao público, traduzindo-as para as práticas do cotidiano dos serviços e tornando-as compreensíveis (ZOMBINI; PELICIONI, 2011).

Quanto às sugestões para o projeto foi apontado aspectos referentes à manutenção e continuidade das ações educativas de forma permanente na região em $66,66 \%$ dos registros $(n=10)$, levando em consideração os impactos do projeto na articulação da rede e no aprimoramento da assistência para os usuários, gestores e profissionais. Outro aspecto apontado como sugestão foi a elaboração de outras cartilhas educativas com informações e orientações sobre os fluxos de redes de atendimento em $20 \%$ dos achados $(n=3)$. Sob essa perspectiva os autores Castro et al., (2017) relatam que a continuidade das ações de projetos vai ao encontro dos princípios da Constituição Federal de 1988, a qual prevê em seu texto do Artigo 207, a indissociabilidade das universidades, ou seja, o alinhamento entre ensino, pesquisa e extensão, fator que contribui à continuidade de ações, permitindo que estes três pilares cooperem de forma efetiva para a sociedade.

\section{CONCLUSÃO}

A assistência ao usuário na rede de saúde, educação e assistência social, de forma interdisciplinar, requer um olhar dos profissionais, das mais diversas áreas, para a subjetividade de cada sujeito, compreendendo assim, sua história, seu lugar na sociedade e nas relações que estabelecem com seus familiares, suas queixas e problemáticas. Assim, ações de educação permanente voltadas para o desenvolvimento de ações intersetoriais 
são de fundamental importância para aprimorar os processos de trabalho entre os diversos setores envolvidos na atenção aos usuários e aperfeiçoar a assistência.

Além disso, avaliar as atividades, etapas e contribuições de projetos, programas e ações possibilita repensar a aplicabilidade das ações e dos recursos, permitindo a reformulação ou aperfeiçoamento dos mesmos, a fim de qualificar o cuidado. Dessa forma, o caráter coletivo das ações de planejamento e desenvolvimento das atividades do projeto foram ao encontro da importância de ouvir os envolvidos na implementação e desenvolvimento da Rede de atenção às PcDs na região, uma vez que, permitiu aprimorar e ampliar o olhar frente às necessidades singulares das pessoas com deficiência, familiares e profissionais, auxiliando para qualificar e elaborar novas ações frente a estas demandas.

Os participantes do projeto avaliaram como muito positivo o desenvolvimento do projeto na região e apontaram suas importantes contribuições para o aprimoramento de suas práticas cotidianas de trabalho e de assistência na rede de atenção às pessoas com deficiência. Entre as contribuições apontadas encontram-se maior clareza nos significados da deficiência, aprimoramento das ações de acolhimento dos PcDs e seus familiares nos serviços da rede de atenção, bem como melhorias na articulação da mesma, com fluxos e encaminhamentos mais claros e efetivos. Outra contribuição mencionada foi a sensibilização dos participantes para a temática, trazendo a importância de um olhar atento e menos excludente a esse segmento. Destacaram também o enfoque interdisciplinar, multiprofissional e interinstitucional do projeto como um dos fatores que contribuiu para o sucesso das ações.

Assim, evidencia-se que o cuidado com as pessoas com deficiência requer constante reflexão acerca dos contextos sociais em que os sujeitos se constituem, bem como das práticas de atenção e cuidado profissional desenvolvidas pelos setores de saúde, educação e assistência social. Nesse sentido, ações de educação permanente, voltadas ao cuidado às pessoas com deficiência, se fazem importantes ferramentas do fazer profissional.

\section{REFERÊNCIAS}

ALMEIDA FILHO, Naomar de. Intersetorialidade, transdisciplinaridade e saúde coletiva: atualizando um debate em aberto. Revista de Administração Pública, Rio de Janeiro, v.34, n.6, p.11-34, 2000.

ALVES, Maria Angélica; RIBEIRO, Fabiane Ferreira; SAMPAIO, Rosana Ferreira. Potencial de mudança nas práticas de saúde: a percepção de trabalhadores de uma Rede de Reabilitação em (trans)formação. Fisioter. Pesqui., São Paulo, v. 23, n. 2, p.185-192, 2016.

AMORIM, Érico Gurgel; LIBERALI, Rafaela; MEDEIROS NETA, Olivia Morais. Avanços e desafios na atenção à saúde e pessoas com deficiência na atenção primária no Brasil: uma revisão integrativa. Holos, Rio Grande do Norte, v. 1, ano 34, 2018.

BARDIN, Laurence. Análise de conteúdo. 1.ed. Lisboa: Edições 70, 2016.

BOURGUIGNON, Jussara Ayres. Concepção de rede intersetorial, 2001. Disponível em: http://www.uepg.br/nupes/intersetor.htm. Acessado em: 07 de março de 2020.

BRASIL, Ministério da Saúde, Secretaria de Políticas de Saúde. Políticas de Saúde: Metodologia de Formulação, 1998. Disponível em: http://bvsms.saude.gov.br/bvs/publicacoes/capa_politicas.pdf. Acessado em: 07 de março de 2020. 
CAMPOS, Kátia Ferreira Costa; MARQUES, Rita de Cácia; CECCIM, Ricardo Burg; SILVA, Kênia Lara. Educação Permanente em Saúde e modelo assistencial: correlações no cotidiano de serviço na Atenção Primária à Saúde. APS em Revista, Belo Horizonte, v.1, n.2, p.132-140, 2019.

CAMPOS, Maria Fernandes; SOUZA, Luiz Augusto de Paula; MENDES, Vera Lúcia Ferreira. A rede de cuidados do Sistema Único de Saúde à saúde das pessoas com deficiência. Interface, Botucatu, v.19, n.52, p.207-210, 2015.

CARMO, Michelly Eustáquia do; GUIZARDI, Francini Lube.Desafios da intersetorialidade nas políticas públicas de saúde e assistência social: uma revisão do estado da arte. Physis Revista de Saúde Coletiva, Rio de Janeiro, v.27, n.4, p.1265-1286, 2017.

CASTRO, G.A. et al. A importância da Extensão na Formação Acadêmica dos Alunos do Curso de Engenharia Civil da PUC Minas Barreiro. In: SANABIO, D.; BARROS, E.B.R.(Org) Extensão PUC Minas: sujeitos, espaços e tempo de ação. Belo Horizonte: PUC Minas, 2017.

DANTAS, K. O. et al. Repercussões do nascimento e do cuidado de crianças com deficiência múltipla na família: uma metassíntese qualitativa. Cad. Saúde Pública, Rio de Janeiro, v. 35, n. 6, p. 1-19, 2019.

MARCON, S.S. et al. Dificuldades e conflitos enfrentados pela família no cuidado cotidiano a uma criança com doença crônica. Cienc Cuid Saude, Maringá, v. 6, n. 2, p. 411-19, 2007.

DIAS, M.S.A. et al. Colaboração interprofissional no Projeto Saúde e Prevenção na Escola. Ciênc. saúde coletiva [online], v.21, n.6, p.1789-1798, 2016.

DUBOW, Camila; GARCIA, Edna Linhares; KRUG, Suzane Beatriz Frantz. Percepções sobre a Rede de Cuidados à Pessoa com Deficiência em uma Região de Saúde. Saúde Debate, Rio de Janeiro, v.42, n.117, p.455-467, 2018.

FRANÇA, I.S.X. et al. 0 descuidar do lesado medular na atenção básica: desafios bioéticos para as políticas de saúde. Rev. bras. enferm., v.65, n.2, p.236-243, 2012.

GOULART, Camila Toczk; BRUN, Adriane Buhrer. A intersetorialidade da Política de urbanismo em relação à garantia de direitos da pessoa com deficiência. Caderno Humanidades em Perspectivas, Curitiba, v.3, n.2, p.3041, 2018.

HACK, Neiva Silvana; CARVALHO, Deborah Ribeiro. A Relação das Políticas de Educação, Saúde e Assistência Social Frente aos Desafios da Educação Inclusiva. Perspectivas em Diálogo: revista de educação e sociedade, v. 5, n. 9, p. 234-252, 2018.

IBGE. Censo Demográfico 2010: Banco de Dados agregados do IBGE. Disponível em: <http://www.ibge.gov.br/home/estatistica/populacao/censo2010/default.shtm>. Acessado em 01 de março de 2020.

IBGE. Diretoria de População e Indicadores Sociais. Estimativas da população residente com data de referência 10 de julho de 2014. Diário Oficial [da] União, 28 ago. 2014. Brasília, 2014. Disponível em: <http://www.ibge.gov.br/home/estatistica/populacao/estimativa2014/estimativa_dou.shtm>. Acessado em 01 de março de 2020.

LAVICH, C.R.P. et al. Ações de educação permanente dos enfermeiros facilitadores de um núcleo de educação em enfermagem. Rev Gaúcha de Enferm, Porto Alegre, v.38, n.1, p.e62261, 2017.

LEMOS, Cristiane Lopes Simão. Educação Permanente em Saúde no Brasil: educação ou gerenciamento permanente? Ciênc. saúde coletiva [online], v.21, n.3, p.913-922, 2016.

MAIA, Evanira Rodrigues; PAGLIUCA, Lorita Marlena Freitag; ALMEIDA, Paulo César de. Learning of community health agent to identify and register disabled people. Acta paul. enferm. v.27, n.4, p. 326-332, São Paulo, 2014. 
MARANHÃO, Governo do Estado, Secretaria de Estado do Planejamento e Orçamento. Manual de Critérios para a Elaboração das Políticas Públicas, 2019. Disponível:https://seplan.ma.gov.br/files/2019/03/Manual-deCrit\%C3\%A9rios-para-a-Elabora\%C3\%A7\%C3\%A3o-das-Pol\%C3\%ADticas-P\%C3\%BAblicas_Formato-Digital.pdf. Acessado em: 07 de março de 2020.

MEIRELLES, Betina Hörner Schlindwein. Redes sociais em saúde: desafio para uma nova prática em saúde e Enfermagem. Trabalho apresentado para Concurso Público ao cargo de Professor Adjunto, Florianópolis: Universidade Federal de Santa Catarina; 2004. disponível em: <http://www.scielo.br/scielo.php?script=sci_arttext\&pid=S0103-21002012000300023> Acessado em: 16 de março de 2020.

ORNELAS, Antonio Lima; TEIXEIRA, Maria Gracinda Carvalho. Intersetorialidade ou diálogos setoriais? Reflexões a partir da experiência do Projeto Teias-Escola Manguinhos, Rio de Janeiro. Saúde Debate, Rio de Janeiro, v.39, n.1, p.659-670, 2015.

PERES, Girlane Mayara; GRIGOLO, Tania Maris; SCHNEIDER, Daniela Ribeiro. Desafios da Intersetorialidade na Implementação de Programa de Prevenção ao Uso Abusivo de Drogas. Psicologia: Ciência e Profissão, Brasília, v.37, n.4, p.869-882, 2017.

SANTOS, R. F. et al. Tecnologia assistiva e suas relações com a qualidade de vida de pessoas com deficiência. Rev. Ter. Ocup. Univ. São Paulo, v. 28, n. 1, p. 54-62, 2017. Disponível em: http://www.revistas.usp.br/rto/article/view/107567/129256. Acessado em: 05 de agosto de 2020.

SERAPIONI, Mauro. Conceitos e métodos para a avaliação de programas sociais e políticas públicas. Revista da Faculdade de Letras da Universidade do Porto, v. 31, p. 59-80, 2016.

SLUZKI, C.E. A rede social na prática sistêmica. São Paulo: Casa do Psicólogo, 1997.

TEDESCO, Janaína dos Reis; JUNGES, José Roque. Desafios da prática do acolhimento de surdos na atenção primária. Cad. Saúde Pública, Rio de Janeiro, v.29, n.8, p.1685-1689, 2013.

TUON, Lisiane; CERETTA, Luciane Bisognin (Org.) Experiências exitosas no âmbito da assistência educacional, da saúde e da assistência social: transversalidade e intersetorialidade. Recurso eletrônico on-line, 2017. Disponível em:

http://repositorio.unesc.net/bitstream/1/5498/1/Experi\%C3\%AAncias\%20exitosas\%20no\%20\%C3\%A2mbito \%20da\%20assist\%C3\%AAncia\%20educacional....pdf. Acessado em: 16 de março de 2020.

ZOMBINI, Edson Vanderlei; PELICIONI, Maria Cecília Focesi. Estratégias para a avaliação de um material educativo em saúde ocular. Rev. Bras. Cresc. e Desenv. Hum., v.21, n.1, p.51-58, São Paulo, 2011. 